\title{
Performance Comparison Between Direct and External Modulation Using RZ and NRZ Coding
}

\author{
Bakir Hadj Ali \\ University of Chlef, Algeria
}

Corresponding author: h.bakir@univ-chlef.dz

\begin{abstract}
Such as in any optical communication system, the light modulation is considered a main key to determine the performance (or the maximum) of the data rate that can be transmitted and over what the long distance can be applied. So, a choice of the modulation type in any optical link is more important and should base on measuring of the BER and the Q factor parameters. In that issue, we propose a comparative study as contribution between the principle or the schemes of the direct modulation (DM) and the external modulation (EM) respectively, which the chirp frequency modeling is described for the two modulations. However, the main issues addressed also in the simulation analysis when the amount of the chirp (in GHz) introduced for the two modulations applied is discussed, and also the Q factor introduced using different power values and, in different data rate for the two modulations studied is presented where we can determine from these results of the Q factor the effect of the power and the limit of applying the direct modulation and the external modulation in terms of the maximum of the data rate that can be transmitted. Moreover, we provide a numerical results of the Q factor and the BER for the two modulations (DM and EM) using RZ and NRZ format codes at different distance transmission, and in different data rate through these results we can conclude in which code (RZ and NRZ) the DM and the EM performed better.
\end{abstract}

Keywords: Intensity modulation, direct modulation, external modulation, MZM modulator, chirp frequency

Article History: received 1 December 2019; accepted 10 March 2020; published 25 April 2020.

(C) 2020 Penerbit UTM Press. All rights reserved

\section{INTRODUCTION}

The rapid evolution of the high data rate in the recent optical systems which the optical fiber may be extent to achieve the users in the FTTH technology, so the optical fiber doesn't still to use connecting networks with each other.

Furthermore, in the most optical links the modulation technique is considered the key to define their performance. While, the modulation used in the optical systems is divided into two types. One is known the intensity direct modulation [1] is obtained when the RF (electrical signal or message) is applied directly to the opto-electronique component either laser or LED diode, which the LED diode in the optical system is reserved to applications needed low performance in data transmitting and applied generally for the short distance. the advantages presented by the intensity of direct modulation are the simplicity, a compactness, but an undesired chirp phenomenon is accompanied this intensity modulation, occurred as rapid change in the centre wavelength of the signal [2-3] due to the strong coupling between the index refractive of the laser cavity and the flux carrier density passed throughout. So, adding to others limitations the DM suffers in any communication system by the chromatic dispersion, and non-linearity effect. There is a chirp phenomenon referred to broadening pulses caused in the direct modulation, it considered a serious problem to limit the performance of the DM especially in the high data rate, and in the long distance. However, for many applications as DWDM (Dense Wavelength Division Multiplex) the chirp can introduce a crosstalk between the wavelengths [4].

To overcome this limitations of the chirp in the intensity of the direct modulation. There has been an alternative solution was external modulations that having less frequency chirping and this EM is divided into 3 classes [1]: intensity modulation; phase modulation, frequency modulation; they designed usually by the MZM modulator or by the electro-absorption modulator. Many researches have reported the performance and a characterization of the intensity modulation used the MZM modulator $[2,5]$. And they have shown that the intensity modulation using MZM modulator can present some advantage [4-5] such as a good speed, handling under low drive voltages, give better extinction ratio. And the waveguide interferometer (component of the MZM) fabricated via Lithium niobate can present low loss and his coupling with optical fiber having $2.5 \mathrm{~dB}$ losses which that value has shown in the laboratory [5].

To contribute to a studies already presented of the intensity modulation, we present in this work a comparison between the performance of the intensity modulation based on the direct modulation and a performance of the intensity modulation (EM) based on the wave guide interferometer of the MZM component while the process of generation the signal modulated using the two modulations is given in the section .2 and in the same section we have described the equations and modeling of the frequency chirping phenomenon caused in two modulations proposed. Then, 
in the section. 3 we will present the model simulated of the optical link based on the DM and on the EM respectively. Followed by a presentation and discussion of the results achieved in term of Q factor and BER parameter that have obtained in the eye diagram analyzer after the simulation of the two model proposed.

Which the chirp frequency (in $\mathrm{GHz}$ ) has measured and his effect has explained for the two modulations (DM, and EM) where we used the lower bit rate [2 Gbs to 4Gbs] for the DM and the high bit rate [15 Gbs to $19 \mathrm{Gbs}$ ] for the EM. Moreover, the effect in performance of the EM (Q and BER) is presented at different values of the ER parameter (parameter of the MZM modulator). Then, the performance of the DM and the EM have provided in term of the $\mathrm{Q}$ value, we simulated the $\mathrm{DM}$ for the data rate [2Gbs to $5 \mathrm{Gbs}$ ] and the EM for the data rate [5Gbs, $10 \mathrm{Gbs}$, $15 \mathrm{Gbs}$ ] the $\mathrm{Q}$ factor curves has presented for the two modulations at various value of power, using the two distance transmission $30 \mathrm{~km}$ and $50 \mathrm{~km}$ respectively. By the results achieved, we have carried out the reason of applied the DM to transmit lower data rate and applied the EM generally to transmit high data rate and impact of power over the two optical links has discussed. The last results, it is dedicated to analysis in which code $\mathrm{RZ}$ or the NRZ code the two modulations could provide a good performance in ( $Q$ value and BER parameter), the results will present numerically from the two tables (see table 1and table 2 in simulation section). Finally, the paper will finish by a conclusion about all what has presented in theory and results simulation.

\section{MODELING SYSTEM}

There are two modulation classes used in the optical communication system.

\subsection{The direct modulation (DM)}

The transmitted signal generated from the direct modulation is shown in the following scheme:

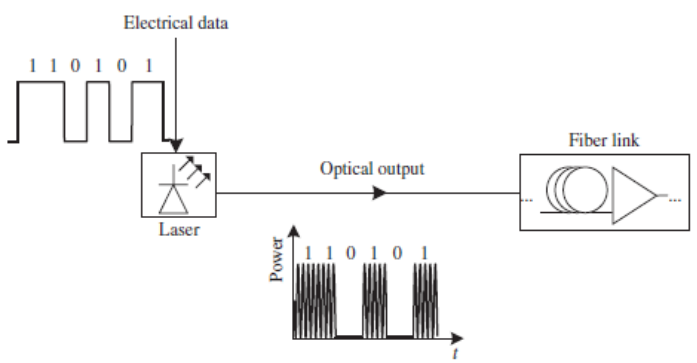

Figure 1. The Direct modulation of semiconductor laser [1]

As shown in the figure 1, the electrical data modulate the drive current of the laser, when the " 1 " of electrical data is present, the laser is turned on and, therefore, the electrical impulsion is encoded onto the presence of optical signal in the fiber link. This modulation present a great amount of chirp that defined as a rapid variation in the instantaneous frequency of the laser caused by the refractive index changes in the active layer due to the carrier density population [6]. Where the chirp and dispersion effect of the fiber will introduce pulse broading.
For that, the performance of this modulation class still limited for data rate $(<10 \mathrm{~Gb} / \mathrm{s})$ and short distance [1].

\subsubsection{Chirp frequency for the DM}

When the bias current is applied to the laser diode generating the direct intensity modulation. This leads to a change in the carrier concentration and also generates in the laser active region some variation in the refractive index. A change in the refractive index is given as [7]:

$\Lambda=\frac{m \lambda}{2 n} ; \quad n$ : is the refractive index

Thus, the laser intensity modulation is generated with undesired phenomenon known chirp frequency (or a change in the wavelength of the laser).

This chirp may be described by the following equation:

$$
\Delta v(t)=\frac{\alpha}{4 \pi}\left(\frac{d}{d t}\left[\ln P_{e}(t)\right]+\kappa P_{e}(t)\right)
$$

Where:

$\Delta v(t):$ is the laser instantaneous frequency.

$\alpha$ : is called the line enhancement factor.

$\kappa$ : is kown as the adiabatic chirp coefficient.

$P_{e}(t)$ : is the laser output power.

And we can see that the chirp consists by two terms:

$1^{\text {st }}$ term: is called the transient chirp, it exist if the transmitted power vary with time.

$2^{\text {nd }}$ term: directly proportional to the power, called the adiabatic chirp, when the different emission frequencies observed under steady state while a 'mark' or 'space' is emitted.

The equation (1) was described just for the fabry-Perot (FP) lasers. And can used as approximation for the other structure lasers (as FDB) [7].

\subsection{The external modulation (EM)}

The second modulation type known as external modulation it is applied by using an external component to modulate the optical signal as given in the following figure:

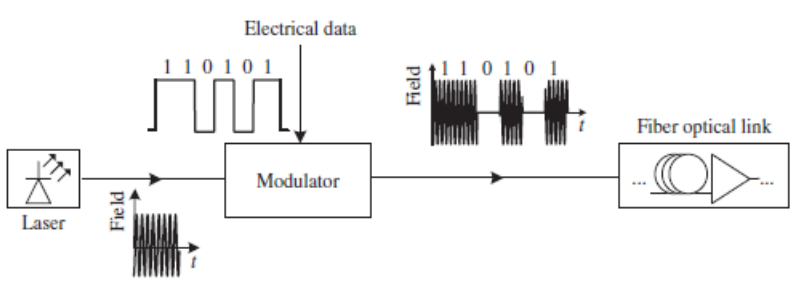

Figure 2. A transmitter using an external modulator [1]

As shown in the figure 2, the laser is turned on at all the time, and we used as external modulator component the MZM modulator having two wave guides, two junctions, and RF/DC traveling wave electrode as shown on the figure 3 .

Figure 3 shows that the external component consist of two waveguide constructed by titanium which is implemented into the lithium niobate substrate, it has as characteristics electro-optic effect. 

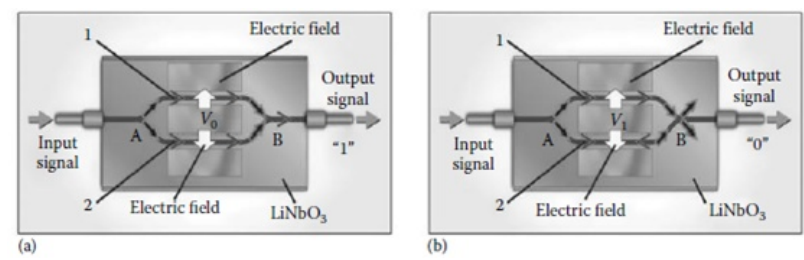

Figure 3. Intensity modulation using interferometric principles (MZM modulator) in guide wave structures in $\mathrm{LiNbO}_{3}$. (a): Constructive interference mode (bit:01), (b) destructive interference mode -(bit:0). Optical guided wave paths 1 and 2. Electric field is established across the optical waveguide [8]

We consider the laser wave launched into MZM modulator as:

$$
E_{\text {in }}(t)=E_{0}(t) e^{-\left(i 2 \pi f_{c} t\right)}
$$

And $f_{c}$ : is the carrier optical frequency.

At the 1-st Y junction, this electrical field is splits into two electrical field of equal power. Where the electrical field passing the upper and lower wave guide is:

$$
E_{j}(t)=\frac{E_{0}}{\sqrt{2}} e^{\left(-i 2 \pi f_{c} t\right)}
$$

Which, $j=1,2$ indicates the two arms of MZM waveguide.

The presence of the factor $\frac{1}{\sqrt{2}}$ in this equation means that the power is reserved across interferometer component.

At the 2-nd Y junction, the component MZM used in the simulation will introduce $E_{\text {out }}$ given as $[9,10]$ :

$$
E_{\text {out }}(t)=E_{\text {in }}(t) \cdot \cos (\Delta \theta(t)) \cdot e^{j \cdot \Delta \varphi}
$$

Which $\Delta \theta$ is the phase difference between the two branches and is defined as:

$$
\Delta \theta(t)=\frac{\pi}{2}(0.5-E R(s(t)-0.5))
$$

$\mathrm{s}(\mathrm{t})$ is the electrical data signal.

ER represents the extinction ratio which it is analyzed in this section. :

$\Delta \varphi$ : is the variation of the signal phase defined as $[9,10]$ :

$$
\Delta \varphi=\frac{S C \cdot \Delta \theta(t) \cdot((1+S F)}{(1-S F)}
$$

The parameter SC is -1 if the negative signal chirp is true or 1 if the negative signal chirp is false. And SF: is the symmetry factor using to characterize the amount of the chirp where it is arising when we increase the value of SF from -1 to 1 [2].

\subsubsection{Chirp frequency for the EM}

This phenomenon results much more in the direct modulation, to describe this parameter we consider the electric field of lightwave whose the carrier optical frequency is $f_{c}$ can be given by [11]:

$$
E(t)=\mathfrak{R}\left[E_{0}(t) e^{i 2 \pi f_{c} t}\right]
$$

And $\mathfrak{R}$ referred to the real part, and $E_{0}(t)$ define the complex envelope of the electric field, which the complex envelope can be written as:

$$
E(t)=|E(t)| e^{i \varphi(t)}=\sqrt{P(t)} e^{i \varphi(t)}
$$

Or $|E(t)|$ and $\varphi$ are the modulus of the complex envelope and the phase respectively.

Such as, the intensity modulation proposed in this study using the external modulation of the MZM that consists of $P(t)$ variation according to the modulating electrical signal of the NRZ considered. But in the signal intensity modulated appear variation of the phase $\varphi(t)$ that considered as undesired parameter. By using the equation (eq.7) and (eq.8), and after we suppose the pulsation $\omega_{0}=$ $2 \pi f_{c} t$, it will be after the chirp phenomenon [11]:

$$
\omega(t)=w_{0}+\frac{\partial \varphi}{\partial t}
$$

As shown from the above equation. The phase variation depends in the time is equivalent to frequency variation referred as frequency chirping that affet much more the direct modulation performance which limit it to use only for the low data rate compared to the external modulation having less this phenomenon as shown in the simulation section.

\subsubsection{Extinction ratio}

Such as after the splitting power presented by the optical field given in the equation (eq.3), at the $1^{\text {st }} Y$ junction of the MZM waveguide, the power coming through two arms may not be equal $1 / 2: 1 / 2$ due to some fluctuation induced in the MZM [1]. And as extinction ratio (ER) defines the ratio of maximum to minimum power. To analyze the ER parameter considering the output optical power at the $2^{\text {nd }}$ junction of the MZM interferometer given by [1]:

$$
\begin{aligned}
P_{\text {out }}=\frac{P_{0}}{\left(\alpha_{1}+\alpha_{2}\right)} & {\left[\alpha_{1}{ }^{2}+\alpha_{2}{ }^{2}\right.} \\
& \left.+2 \alpha_{1} \alpha_{2} \cos \left(\varphi_{1}-\varphi_{2}\right)\right]
\end{aligned}
$$

Which $\alpha_{1}{ }^{2}+\alpha_{2}{ }^{2}=1$; for ideal case about splitting power $\alpha_{1}=\alpha_{2}=\frac{1}{\sqrt{2}}$.

Taken the two following cases of summing between two optical waves passing the two arms that having $\varphi_{1}, \varphi_{2}$ as phases respectively, we can understand in which case the MZM lead to transmit bit " 1 " as shown in the output of the MZM presented in the point B (figure 3, a) and in which case the MZM lead to transmit bit ' 0 ' as shown in the output of the MZM presented in the point B (figure 3, b). 
Case 1: when $\varphi_{1}-\varphi_{2}=0$, the interference is constructive and the output is maximum as given the equation (eq. 10) and as shown in the figure 4 :

$P_{\text {out }}^{\max }=P_{0}$

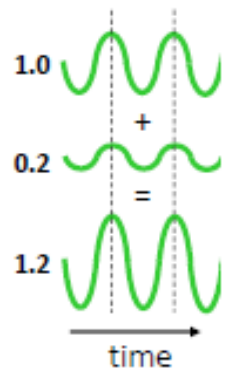

Figure 4. Constructive interference [12]

Case 2: when $\varphi_{1}-\varphi_{2}=\pi$, the interference is destructive and the output is minimum and is given from the equation (eq. 10) and for understanding this case we present figure 5:

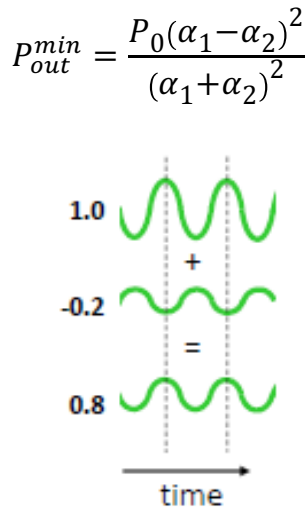

Figure 5. Destructive interference [12]

So, the ER parameter is defined as:

$$
E R=\frac{P_{\text {out }}^{\max }}{P_{\text {out }}^{\min }}=\left(\frac{\alpha_{1}+\alpha_{2}}{\alpha_{1}-\alpha_{2}}\right)^{2}
$$

Which this ER parameter is done generally in $\mathrm{dB}$ unit and can be written as:

$$
E R(\text { in } d B)=10 \log _{10} E R
$$

\section{Simulation results}

\subsection{The optical links simulated}

In this section, we present the model of the optical link simulated from applying the direct and the external modulation and we anlysis the simulation results acheived. While, we generate the binary data sequence that encoded then by the NRZ or RZ pulse generator. These NRZ and $\mathrm{RZ}$ electrical signals are injected to the laser diode for the DM, secondly the NRZ, RZ signals are injected to the MZM component for the EM to modulate it into the optical domain with the power generated by $\mathrm{CW}$ diode laser.

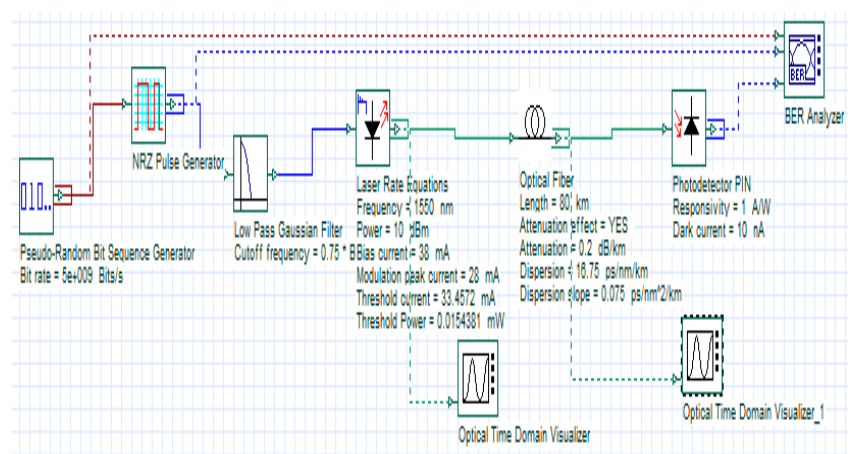

Figure 6. The model simulated for the direct modulation

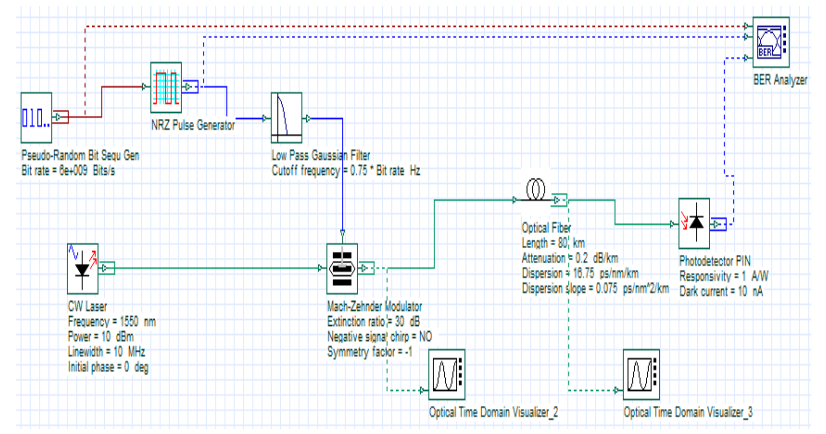

Figure 7. The model simulated for the external modulation using Mach-Zehnder Modulator

\subsection{The result of the chirp and $Q$ factor}

In the two following figures (figure 8 and figure 9). We have presented firstly the evolution of the chirp frequency introduced in the optical link based on the direct modulation simulated as shown in the figure 8 after we varied the data binary from 2 Gbs to 4 Gbs. Secondly, in the figure 9 we have presented a relation between the chirp curve and $Q$ factor when we varied always the data binary ( $2 \mathrm{Gbs}$ to $4 \mathrm{Gbs}$ ). The laser power is fixed at $10 \mathrm{dBm}$ and the distance simulated is $20 \mathrm{~km}$.

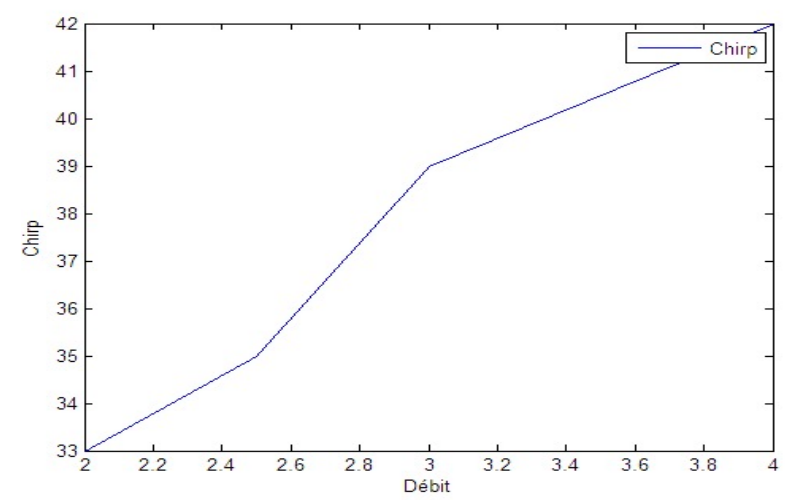

Figure 8. Chirp phenomenon $(\mathrm{GHz})$ of the direct modulation versus different data binary (in $\mathrm{Gb} / \mathrm{s}$ ) 


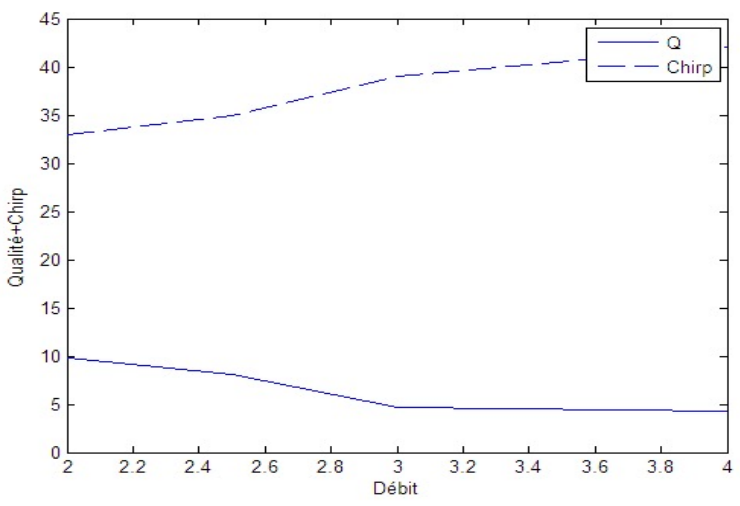

Figure 9. Chirp amount (in $\mathrm{GHz}$ ) and factor $\mathrm{Q}$ versus different data binary $(\mathrm{Gb} / \mathrm{s})$ for the direct modulation

In the figure 8 , it can be seen that the frequency chirp (or known as broadening impulsion) measured is great and it is increased from $(33 \mathrm{GHz}$ to $42 \mathrm{GHz}$ ) when we increased the data binary from the interval of [ $2 \mathrm{Gbs}$ to $4 \mathrm{Gbs}$ ]. And this increasing of the chirp frequency has introduced decreasing in the $\mathrm{Q}$ factor of the DM especially when the data binary is greater than $3 \mathrm{~Gb} / \mathrm{sec}($ where $\mathrm{Q}<6$ ) as shown from the figure 9. However, this effect is greater in the direct modulation [2, 8]. Which more chirp frequency means more broadening impulsions transmitted and more interference introduced between the symbols in the reception side.

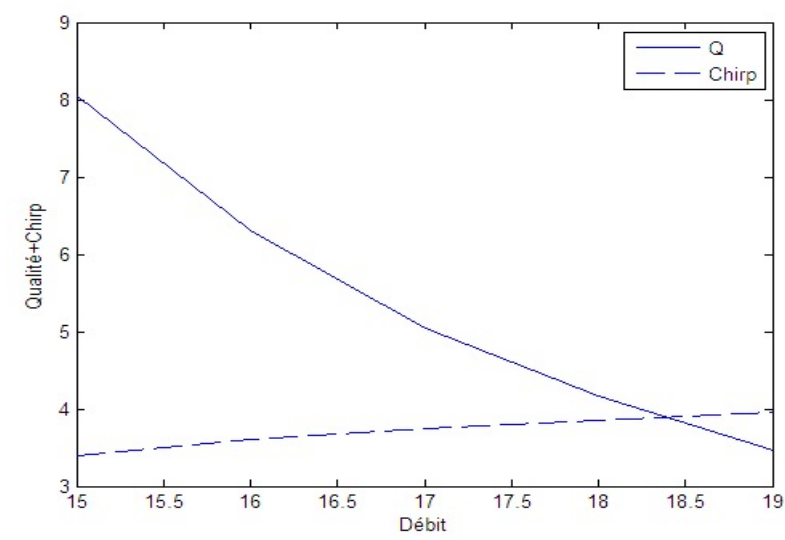

Figure 10. chirp amount (in $\mathrm{GHz}$ ) and factor $\mathrm{Q}$ versus different data binary (in $\mathrm{Gb} / \mathrm{s}$ ) for the external modulation

From the results of the Q value and amount of the chirp (in $\mathrm{GHz}$ ) as plotted in the figure 10. It can be observed, increasing the data simulated from [ $15 \mathrm{Gbs}$ to $19 \mathrm{Gbs}$ ] less effect has introduced about the chirp while the amount of the chirp has increased from [3.5 GHz to $4 \mathrm{GHz}$ ] or the EM has limited the phenomenon of the chirp in the high bit rate $[1,2]$. In the same figure, we have seen a great influence has provided about the performance of the EM in term of the $Q$ value. Which, the $Q$ value decreased from [8 to 3.5]. By these results, the EM can transmit until $16.3 \mathrm{Gbs}$ with $\mathrm{Q} \geq 6$, and these performance of the data transmitted considered important by applying the EM.

So, it can be seen that the chirp affect also the performance of the EM in the data rate $>16 \mathrm{Gbs}$, and it can be concluded from this comparison results (relation of the $\mathrm{Q}$ and chirp) that the chirp could produce transmission errors; and it considered as one of the negative effect appeared in the optical communication systems among the other negative effects as dispersion, none linearity of optic fiber and others [13].

\subsection{The effect of the ER for external modulation:}

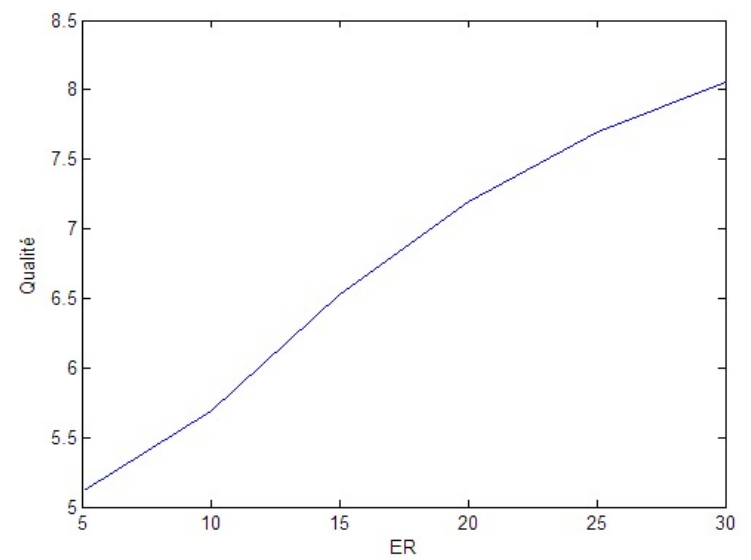

Figure 11. The Q factor versus ER (extinction ratio) parameter

The figure 11 shows the influence of the ER parameter about the component of the Mach-zehnder modulator used in the EM link. While, the performance of the EM is measuring always in term of the Q value. From the curve plotted in figure.11, a great enhancement in the EM has introduced as ER parameter increased. Furthermore, the EM is considered well in term of the data transmitted if ER $>12$ (that value can produce $\mathrm{Q}>6$ ).

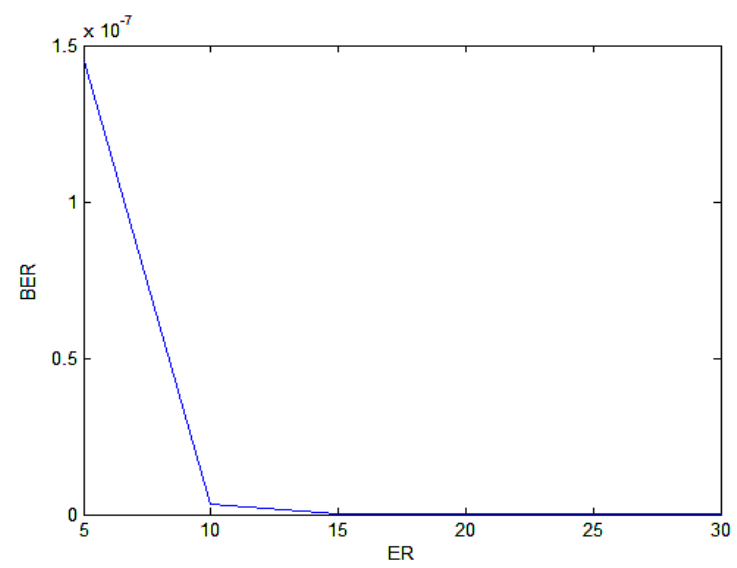

Figure 12. The Bit Error Rate (BER) versus ER ratio

In the figure 12, we have shown the BER parameter obtained after we varied the ER (from 5 to 30). A great improvement appeared in term of the BER of the EM when we increased the ER parameter in the simulation. Such as, a great value of ER ratio facilitate in the reception side to distinguish between the bit ' 0 ' reception and the bit ' 1 ' reception [11] and that make a receiver having a good performance. 


\subsection{The effect of the data binary, the distance and the} power

\subsection{1 for the direct modulation}

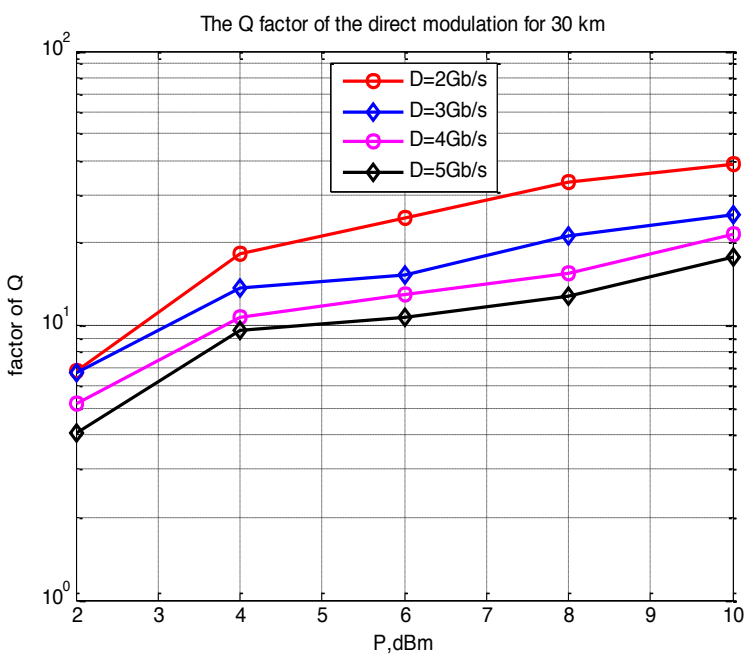

Figure 13. The Q performance of the direct modulation in various data speed $(\mathrm{Gb} / \mathrm{s})$ simulated in different values of $\mathrm{P}(\mathrm{dBm})$ using $30 \mathrm{~km}$ distance

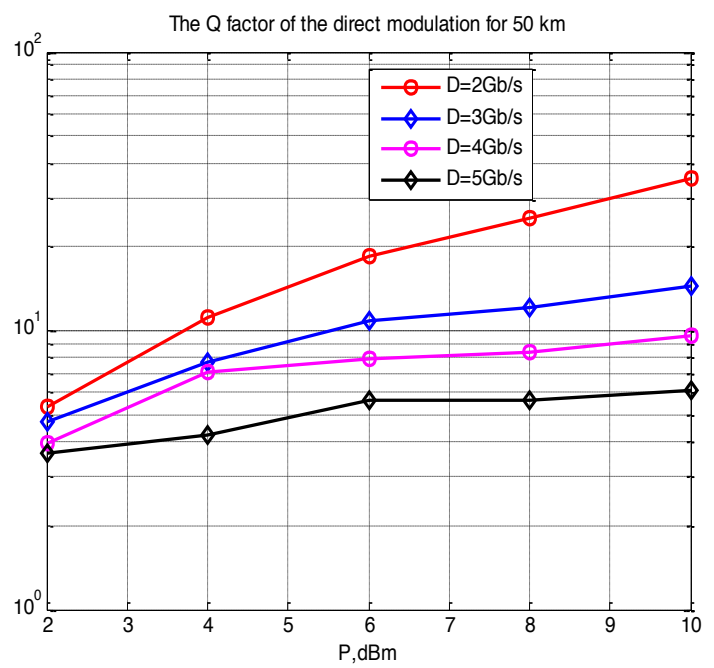

Figure 14. The Q performance of the direct modulation in various data speed $(\mathrm{Gb} / \mathrm{s})$ simulated in different values of $\mathrm{P}(\mathrm{dBm})$ using $50 \mathrm{~km}$ distance

From the figure 13 and figure 14 , it can be seen that increasing the data transmitted from $2 \mathrm{Gbs}$ to $5 \mathrm{Gbs}$ introduced decreasing in the performance of the DM presented by the $\mathrm{Q}$ factor. And this performance is decreased more when we increased the distance transmission from $30 \mathrm{~km}$ to $50 \mathrm{~km}$.

Other observation about the effect of the power, the performance of the $\mathrm{Q}$ is improved when we increased the power of the laser diode used in the simulation. But having less effect in the $\mathrm{Q}$ factor at $50 \mathrm{~km}$ distance and for the $3 \mathrm{Gbs}$, 4Gbs and $5 \mathrm{Gbs}$ data successively. So, the power couldn't recompense the broadening of the impulsion resulted in the $50 \mathrm{~km}$ when we used in the simulation 3 Gbs to 5 Gbs.

We limited the simulation at $5 \mathrm{Gbs}$ because after we simulated the bit rate $6 \mathrm{~Gb} / \mathrm{s}$ with $30 \mathrm{~km}$ and up to $10 \mathrm{dBm}$ as power we obtained 4.97 for the $\mathrm{Q}$ factor and the accepted Q in the optical transmission is 6 [2]. Then, the direct modulation can apply for the data speed less than $(\leq$ 5 Gbs).

Consequently, the performance of the DM is decreased when we increased either the data or the distance which the chirp phenomenon is increased if the data simulated is increased.

\subsection{2 for the external modulation}

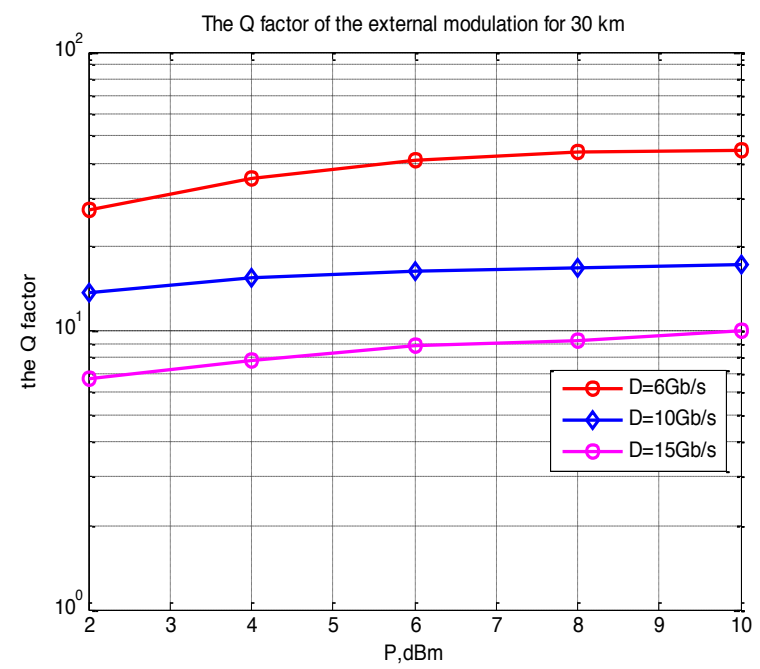

Figure 15. The Q performance of the external modulation in various data speed $(\mathrm{Gb} / \mathrm{s})$ simulated in different values of $\mathrm{P}(\mathrm{dBm})$ using $30 \mathrm{~km}$ distance

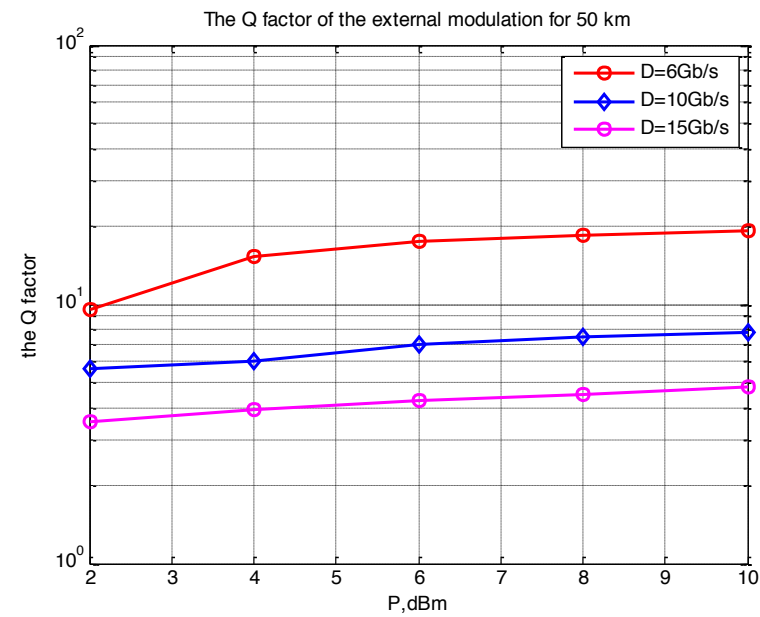

Figure 16. The Q performance of the external modulation in various data speed $(\mathrm{Gb} / \mathrm{s})$ simulated in different values of $\mathrm{P}(\mathrm{dBm})$ using $50 \mathrm{~km}$ distance

As shown in the figure 15 and figure 16, we have simulated the 2nd optical link proposed that based on the external modulation for the data rate of $6 \mathrm{Gbs}, 10 \mathrm{Gbs}, 15 \mathrm{Gbs}$ respectively and we have used $30 \mathrm{~km}, 50 \mathrm{~km}$ respectively. So, in the curves those have given in the figure 15 and figure 16 . We have observed that the EM performance decreased as the data rate increased at 30 and $50 \mathrm{~km}$. because the impulsion duration of the NRZ code used in simulation decreased when the data rate increased this will generate in receiver more probability to interferences between symbols encoded that interpreted by more errors (which less $Q$ has obtained). For increasing of the 
transmission distance (30 $\mathrm{km}$ to $50 \mathrm{~km}), \mathrm{EM}$ performance has decreased (exemple: with $\mathrm{D}=10 \mathrm{Gbs}, \mathrm{P}=4 \mathrm{dBm}$; at $\mathrm{L}=30 \mathrm{~km}, \mathrm{Q}=18$ but at $\mathrm{L}=50 \mathrm{~km}, \mathrm{Q}=6$ ). So, the performance of the EM has decreased because more distance means more broadening in the NRZ impulsion (generated by chirp and dispersion of the optical fiber) this will give in receiver more probability to interference among the impulsion transmitted.

About the power effect, it can be seen that no important improvements of the EM performance has introduced by increasing the power in the simulation from $(2 \mathrm{dBm}$ to $10 \mathrm{dBm})$ for all the data rate simulated and for the two distances used $(30 \mathrm{~km}$ and $50 \mathrm{~km})$. Compared to great improvements in the DM performance provided by increasing the power (see figure 13 and figure 14). So, the effect of increase the power parameter appeared in the lower data rate (case: optical link of the DM), and no significant of power has given in high data rate (case: optical link of the EM).

Despite this, the EM has given better performance $(Q>6)$ from the external component of the MZM modulator that limit the chirp for all the bit rate simulated (5Gbs, 10Gbs, and 15Gbs) and for the two distances used ( $30 \mathrm{~km}$ and $50 \mathrm{~km}$ ) except that of the bit rate $15 \mathrm{Gbs}$ at 50 $\mathrm{km}$ (or the $\mathrm{Q}$ value $>6$ ) has provided if the power Pin $\geq 4$.
3.5 The performance comparison of the RZ and NRZ codes over the DM and the EM systems

The numerical results of the Q-factor and BER presented in the table 1 and in the table 2 respectively have given to compare the performance of the NRZ and the RZ formats coding across the two modulations proposed (DM and EM). We present in the table 1 the direct modulation performance and in the table 2 the external modulation performance.

For the direct modulation: we have used the data rate 2 and $5 \mathrm{~Gb} / \mathrm{s}$ respectively, and we have increased a distance from $10 \mathrm{~km}$ up to $50 \mathrm{~km}$.

For the external modulation: we have used in this simulation the data rate $10 \mathrm{~Gb} / \mathrm{s}$ and $15 \mathrm{~Gb} / \mathrm{s}$ respectively. While, the distance has increased from $10 \mathrm{~km}$ up to $50 \mathrm{~km}$.

It is noted in the table 1 about the DM, the NRZ signals have a higher $\mathrm{Q}$ factor and lower BER that means a better performance has obtained in the DM by using the NRZ signals compared to $\mathrm{RZ}$ signals.

In contrast, from the table 2 about the EM the RZ signals have presented better performance in the $\mathrm{Q}$ factor and in the BER compared to results of the $\mathrm{Q}$ factor and the BER provided by the NRZ signals.

\begin{tabular}{|c|c|c|c|c|c|c|c|c|}
\hline \multicolumn{4}{|c|}{ NRZ } & \multicolumn{4}{c|}{ RZ } \\
\hline \multirow{2}{*}{$\begin{array}{c}\text { Distance } \\
(\mathbf{k m})\end{array}$} & \multicolumn{2}{|c|}{$\mathbf{2 ~ G b / s}$} & \multicolumn{2}{|c|}{$\mathbf{5 ~ G b / s}$} & \multicolumn{2}{|c|}{$\mathbf{2 ~ G b / s}$} & \multicolumn{2}{c|}{$\mathbf{5 ~ G b} / \mathbf{s}$} \\
\cline { 2 - 10 } & $\mathbf{Q}$ & $\mathbf{B E R}$ & $\mathbf{Q}$ & $\mathbf{B E R}$ & $\mathbf{Q}$ & $\mathbf{B E R}$ & $\mathbf{Q}$ & BER \\
\hline $\mathbf{1 0}$ & 65,63 & 0 & 32,94 & $2,42 \mathrm{E}-238$ & 64,34 & 0 & 16,74 & $3,05 \mathrm{E}-63$ \\
\hline $\mathbf{2 0}$ & 62,46 & 0 & 16,63 & $2,03 \mathrm{E}-62$ & 46,38 & 0 & 11,92 & $4,24 \mathrm{E}-33$ \\
\hline $\mathbf{3 0}$ & 45,05 & 0 & 10,49 & $4,65 \mathrm{E}-26$ & 31,43 & $3,22 \mathrm{E}-217$ & 6,75 & $6,95 \mathrm{E}-12$ \\
\hline $\mathbf{4 0}$ & 33,07 & $2,56 \mathrm{E}-240$ & 8,52 & $7,98 \mathrm{E}-18$ & 26,21 & $7,83 \mathrm{E}-152$ & 3,48 & $2,15 \mathrm{E}-04$ \\
\hline $\mathbf{5 0}$ & 30,32 & $2,27 \mathrm{E}-202$ & 5,83 & $2,72 \mathrm{E}-09$ & 19,66 & $1,94 \mathrm{E}-86$ & 3,59 & $1,23 \mathrm{E}-04$ \\
\hline
\end{tabular}

Table 1. The $Q$ and BER results with the direct modulation using RZ and NRZ coding

\begin{tabular}{|c|c|c|c|c|c|c|c|c|}
\hline \multirow{2}{*}{$\begin{array}{c}\text { Distance } \\
(\mathbf{k m})\end{array}$} & \multicolumn{4}{|c|}{$\mathbf{N R Z}$} & \multicolumn{4}{|c|}{$\mathbf{R Z}$} \\
\cline { 2 - 10 } & $\mathbf{Q}$ & $\mathbf{1 0} \mathbf{~ G b} / \mathbf{s}$ & \multicolumn{2}{|c|}{$\mathbf{1 5} \mathbf{~ G b} / \mathbf{s}$} & \multicolumn{2}{|c|}{$\mathbf{1 0 ~ G b} / \mathbf{s}$} & \multicolumn{2}{|c|}{$\mathbf{1 5} \mathbf{G b} / \mathbf{s}$} \\
\hline $\mathbf{1 0}$ & 37,87 & $1,65 \mathrm{E}-134$ & 21,14 & $1,45 \mathrm{E}-99$ & 53,08 & 0 & 28,28 & $2,06 \mathrm{E}-176$ \\
\hline $\mathbf{2 0}$ & 19,89 & $1,54 \mathrm{E}-88$ & 8,88 & $2,41 \mathrm{E}-19$ & 50,50 & 0 & 9,27 & $8,59 \mathrm{E}-21$ \\
\hline $\mathbf{3 0}$ & 16,59 & $3,73 \mathrm{E}-62$ & 6,77 & $6,05 \mathrm{E}-12$ & 19,27 & $3,59 \mathrm{E}-83$ & 6,78 & $5,22 \mathrm{E}-12$ \\
\hline $\mathbf{4 0}$ & 9,81 & $4,46 \mathrm{E}-23$ & 3,7 & $7,47 \mathrm{E}-05$ & 15,32 & $2,36 \mathrm{E}-53$ & 5,9 & $1,75 \mathrm{E}-09$ \\
\hline $\mathbf{5 0}$ & 6,02 & $7,63 \mathrm{E}-10$ & 2,59 & $4,61 \mathrm{E}-03$ & 10,06 & $3,48 \mathrm{E}-24$ & 4,73 & $1,05 \mathrm{E}-06$ \\
\hline
\end{tabular}

Table 2. The $\mathrm{Q}$ and BER results with external modulation using RZ and NRZ coding

\section{CONCLUSION}

In this paper, we have investigated the process of a generation the light modulation through the direct modulation (DM) and the external modulation (EM) respectively. Furthermore, the chirp frequency $(\mathrm{GHz})$ has described for the two modulations proposed. The 
important issues provided has carried out from the simulation results when the two optical links proposed have simulated basing on varying the power Pin, the bit rate, the transmission distance, and applied the two codes RZ and NRZ in the simulation. Based on the results obtained, it can be concluded the following observation: The chirp has considered the main factor to determine the performance of the two modulations presented, where a great amount of the chirp ( $33 \mathrm{GHz}$ to $42 \mathrm{GHz}$ ) has introduced for the DM in the bit rate [2 $\mathrm{Gbs}$ to $4 \mathrm{Gbs}$ ]. In contrast, less amount of the chirp $(3.3 \mathrm{GHz}$ to $4 \mathrm{GHz})$ has obtained for the EM in the bit rate [15 Gbs to $19 \mathrm{Gbs}$ ] and for that reason the EM modulation has presented a better performance as compared to the DM while the EM is usually used to transmit high capacity of the data rate.

As we have seen, what impact of the ER parameter (parameter of the MZM component) has produced in the EM performance as shown in the figure 12. Furthermore, it has been observed in the performance of the DM that as the signal power increases, $Q$ value increase also up to 10 $\mathrm{dBm}$ for all bit rates (2 Gbs to $5 \mathrm{Gbs}$ ) simulated as plotted in figure 13 and figure 14. But, no further increase in the EM performance could be observed in the range $(2 \mathrm{dBm}$ to $10 \mathrm{dBm})$ for all bit rates (6Gbs, $10 \mathrm{Gbs}$, and $15 \mathrm{Gbs})$ simulated as shown in figure 15 and figure 16

In the last, based on the numerical results of the $Q$ value and the BER presented in the table 1 and table 2, the RZ and NRZ performed differently for the two modulations used. Which the NRZ has considered best than the RZ code in the DM, but the RZ has considered best than NRZ in the EM. As well as, we concluded that the range of applying the DM can transmit until $5 \mathrm{Gbs}$ at $40 \mathrm{~km}$ using NRZ, and at $30 \mathrm{~km}$ using RZ code. Moreover, the range of applying the EM (provided $\mathrm{Q}>6$ ) can transmit until 15 Gbs at 30 $\mathrm{km}$ using RZ or NRZ respectively.

\section{REFERENCES}

[1] Shiva Kumar and M. Jamal Deen, 'fiber optic communications fundamentals and applications'chapter. $4,1^{\text {st }}$ edn, edition. John Wiley \& Sons, 2014.
[2] Paul O. Idowu1 et al "Comparative Study of Chirp in Direct and External Optical Modulation" International Journal of Computer Science and Information Technology \& Security (IJCSITS), Vol.7, No.2, Mar-April 2017.

[3] G. Morthier, B. Moeyersoon "Improvement of the Direct Modulation Behavior of Semiconductor Lasers by Using a Holding Beam" IEEE Photonics Technology Letters, Vol. 16, NO. 7, July 2004.

[4] C. E. Rogers III, et al "Characterization and Compensation of the Residual Chirp in a MachZehnder-Type Electro-Optical Intensity Modulator" Optical Society of America, Mar 2010.

[5] Charles Cox et al "Techniques and Performance of Intensity-Modulation Direct-Detection Analog Optical Links" IEEE Transactions on Microwave Theory and Techniques, Vol. 45, No. 8, August 1997.

[6] Hamutal Shalom, and al "On the Various Time Constants of Wavelength Changes of a DFB Laser Under Direct Modulation" IEEE Journal of Quantum Electronics, vol. 34, no. 10, october 1998.

[7] P. Krehlik, "Characterization of semiconductor laser frequency chirp based on signal distortion in dispersive optical fiber" Opto-electronics Review 14 (2), 123-128.

[8] Le Nguyen Binh “Optical Fiber Communications Systems: Theory and Practice with MATLAB $®$ and Simulink ${ }^{\circledR}$ Models" Chapter.5, edited by Taylor \& Francis Group, 2015.

[9] Osman WM, Billal KH, Al Nabi AB "Bit Error Rate Performance for Optical Fiber System" J Electr Electron Syst, vol.7, issu.1, 2018.

[10] https://optiwave.com

[11] Christophe Peucheret 'Direct and External Modulation of Light' 34129 Experimental Course in Optical Communication, 2009.

[12] Stavros Iezekiel, "Electrical-to-optical conversion: modulators" Lecture 08, 2015.

[13] Jing Niu, Jianqiao Shen, Niu Yubing“"A Study on the Effects of Dispersion and Nonlinearity in Optical Fiber Communication Systems using DCF" DOI 10.5013/IJSSST.a.17.33.36. 